Original Article

\title{
Effect of sleep posture on neck muscle activity
}

\author{
Won-Hwee Lee, PT, PhD ${ }^{1)^{*}}$, Min-SeOK Ko, PhD²) \\ 1) Department of Physical Therapy, Jeonju Vision College: 235 CheonJam-ro, Wansan-gu, Jeonju-si, \\ Jeollabuk-do 55069, Republic of Korea \\ 2) Department of Public Health Administration, Jeonju Vision College, Republic of Korea
}

\begin{abstract}
Purpose] This study investigated the effect of sleep posture on neck muscle activity. [Subjects and Methods] The study recruited 20 healthy subjects, who were positioned in three supine sleeping positions: both hands at sides, both hands on the chest, and dominant hand on the forehead. The activities of the scalene and upper trapezius muscles bilaterally were measured by surface electromyography. [Results] The upper trapezius and scalene muscle activity on the right side was significantly greater in the supine with dominant hand on the forehead position than in the other positions. [Conclusion] Sleep posture is important and prevent neck and shoulder musculoskeletal pain.

Key words: Electromyography, Neck muscle, Sleep posture
\end{abstract}

(This article was submitted Feb. 17, 2017, and was accepted Mar. 11, 2017)

\section{INTRODUCTION}

Sleep is a mechanism for restoring the body and its functions and maintaining energy and health, and has renewing and replenishing effects both physically and emotionally ${ }^{1)}$. Those who cannot sleep sufficiently tend to have more mood problems, reduced cognitive ability, and increased fatigue and physical discomfort compared with those who sleep normally ${ }^{2}$. The quality of sleep is directly related to human health, as well as to the standard of living. Good-quality sleep is associated with improvements in chronic and acute pain ${ }^{3,4)}$.

The nighttime sleeping posture is strongly related to the quality of sleep ${ }^{5}$. The sleeping posture is related to musculoskeletal disorders of the shoulder or neck, as well as to headaches ${ }^{6}$. Specifically, poor cervical posture during sleep, which is believed to increase biomechanical stresses on the structure of the cervical spine, can produce cervical pain and stiffness, headache, and scapular or arm pain, resulting in low-quality sleep ${ }^{7}$. Although incorrect sleep postures can aggravate pain, the use of an appropriate pillow can relieve neck pain ${ }^{8}$. In the supine positions, normal spinal curvature should be maintained. In side-lying positions, the cervical and thoracic portions of the spine should align with each other so that there is no muscle stiffness and no excessive load imposed on the cervical facet joint ${ }^{2}$. If people with cervical pain use a pillow that supports their neck, their quality of sleep can be enhanced ${ }^{9}$. Therefore, most studies of patients with cervical spine pain have investigated the effects of different pillow types on pain intensity, shape of cervical spine and muscle fatigue ${ }^{2,8,10)}$. Even when using the most comfortable pillow, the quality of sleep and neck muscle activity should differ according to the habitual sleep posture. However, few studies have investigated the effects of various sleep posture on the activity of neck muscles.

To sleep, many people lie in supine or side-lying position ${ }^{2}$. In a supine position, an individual can adopt various postures, such as with both hands positioned at the side (BHS; arms abducted $0^{\circ}$ ), or on the chest (BHC; arms abducted $45^{\circ}$ ), or the dominant hand on the forehead (DHF; dominant arm abducted $90^{\circ}$ ). This study investigated the effects of threes three supine positions on neck muscle activity.

\footnotetext{
*Corresponding author. Won-Hwee Lee (E-mail: whlee@jvision.ac.kr)

(C2017 The Society of Physical Therapy Science. Published by IPEC Inc.

This is an open-access article distributed under the terms of the Creative Commons Attribution Non-Commercial No Derivatives (by-nc-nd) License $<$ https://creativecommons.org/licenses/by-nc-nd/4.0/>.
} 


\section{SUBJECTS AND METHODS}

Twenty healthy volunteers (10 males, 10 females) participated. Their mean age, body mass, and height were $23.1 \pm$ 1.2 years, $58.1 \pm 11.1 \mathrm{~kg}$, and $166.4 \pm 9.8 \mathrm{~cm}$, respectively. All of the participants were right-side dominant. The inclusion criteria were no neck or shoulder musculoskeletal pain and full ranges of cervical and shoulder joint motion. The exclusion criteria were a history of surgery or acute orthopedic injury in the last 6 months. Before participating, the participants provided written informed consent. This study was approved by the Institutional Review Board at Jeonju University (jjIRB150915-HR-2015-0906).

To attain a sleep environment, the laboratory was kept quiet and lights were turned off. To block external stimulation, the participants also wore eye patches and earplugs. The participants lay in the supine position with their head resting on a pillow measuring $37 \times 16 \times 8 \mathrm{~cm}$. The participants adopted one of the three supine positions (i.e., BHS, BHC, and DHF) and held each position for $30 \mathrm{~min}$.

Electromyography (EMG) data were collected using a BTS FreeEMG100RT (BTS Bioengineering, Italy) and analyzed using the EMG Analyzer software (BTS Bioengineering, Italy). A digital band-pass filter (Lancosh FIR) was used to filter movement artifacts $(20-500 \mathrm{~Hz})$. The sampling rate was set at 1,000 Hz. The EMG signals were processed to obtain the root mean square (RMS) using a moving 50-ms window. While the participants maintained each position, EMG signals were recorded for $30 \mathrm{~min}$. The EMG signals from minutes 10 to 20 were used for the data analysis. To minimize skin resistance, the electrode sites were shaved and cleaned with rubbing alcohol. The surface electrodes were fixed on the appropriate sites $^{11)}$. Two electrodes were placed parallel to the upper trapezius and scalene muscle fibers bilaterally. For normalization, the mean RMS of three trials of 40-s reference voluntary contraction (RVC) was calculated for the neck muscles. To measure $\mathrm{RVC}$, the subjects were positioned in a supine position with their arms at their sides without a pillow for 1 min. The order of measurement was randomized according to sleep postures.

The data are expressed as the means and standard deviations. A one-sample Kolmogorov-Sminov test was used to evaluate the normal distribution of the collected data. The significance of differences in EMG muscle activities among the three sleeping postures was assessed using repeated one-way analysis of variance (ANOVA) with the significance level set at 0.05 . The statistical package for the Social Sciences for Window version 18.0 (SPSS, Inc., Chicago, IL, USA) was used for the statistical analysis.

\section{RESULTS}

The activities of the right trapezius and scalene muscles differed significantly in the three sleeping postures $(\mathrm{p}<0.05)$. The activities of the right trapezius and scalene muscles were significantly greater in the DHF position than in the BHS position $(p<0.05)$. The activities of the left trapezius and scalene muscles did not differ significantly in the three sleeping postures (p>0.05; Table 1).

Table 1. Differences in scalene and upper trapezius muscle activities among the three sleeping postures

\begin{tabular}{lll}
\hline Muscle & Posture & Mean \pm SD \\
\hline Right scalene & BHS & $1.00 \pm 0.13$ \\
& BHC & $1.04 \pm 0.19$ \\
Left scalene & DHF & $1.41 \pm 0.64^{*}$ \\
& BHS & $1.00 \pm 0.31$ \\
& BHC & $1.05 \pm 0.43$ \\
Right upper trapezius & DHF & $1.12 \pm 0.70$ \\
& BHS & $0.93 \pm 0.15$ \\
Left upper trapezius & BHC & $0.93 \pm 0.16$ \\
& DHF & $1.92 \pm 1.61^{*}$ \\
& BHS & $0.99 \pm 0.09$ \\
& BHC & $1.02 \pm 0.85$ \\
& DHF & $0.98 \pm 0.64$ \\
\hline
\end{tabular}

BHS: both hands at the side in supine; BHC: both hands on the chest in supine; DHF: dominant hand on the forehead in supine; SD: standard deviation

${ }^{*} \mathrm{p}<0.05$ vs. BHS 


\section{DISCUSSION}

This study investigated the effects of the supine BHS, BHC, and DHF positions on neck muscle activity. The activities of the right trapezius and scalene muscles differed in the three sleeping postures, and the difference between the DHF and BHS positions was significant. There are several reasons for these differences. First, the DHF posture involved greater abduction than the other postures. Glenohumeral joint abduction and flexion occur simultaneously with scapular upward rotation. Assuming a 2:1 scapulohumeral rhythm, shoulder abduction up to about $90^{\circ}$ occurs as the sum of $60^{\circ}$ of glenohumeral abduction and $30^{\circ}$ of scapulothoracic upward rotation ${ }^{12)}$. Therefore, upward rotation of the scapula is an essential component of arm abduction. The function of upper trapezius is scapular upward rotator and the upper trapezius forms a force couple with lower trapezius and serratus anterior ${ }^{12}$. Therefore, the activity of right upper trapezius muscle is increased in the DHF posture. Second, the DHF posture involves $30^{\circ}$ of scapulothoracic upward rotation through a synchronous 20 to $25^{\circ}$ of clavicular elevation at the sternoclavicular joint ${ }^{13}$. The scalene muscle inserts at the first and second ribs and elevates the ribcage $^{14)}$. Therefore, the activity of right scalene muscle is increased in the DHF posture.

The scalene muscle is extrinsic cervical flexors and contributes to forward translation of the cervical vertebrae. The extrinsic muscles of the cervical spine are located farther from the axis of motion and provide power to the motion, but not necessarily precision of motion ${ }^{15}$. The balance of the extrinsic and intrinsic cervical flexors is critical for precise, pain-free motion of the cervical spine. Impairment of the intrinsic cervical flexors has been reported in patients with cervicogenic headache and chronic cervical pain ${ }^{16,17}$. The upper trapezius muscle is an extrinsic cervical extensor that functions to produce extension with posterior translation of the cervical vertebrae ${ }^{15)}$. The pain is typically located in the posterior cervical region with possible radicular symptoms along the cervical nerve root dermatomes or border of the scapular ${ }^{18)}$. Tightness of the upper trapezius can compress the greater occipital nerve, which can cause a tension headache, while tightness of the scalene can cause sensory (tingling, pain, and numbness) and motor (weakness and partial paralysis of the upper extremity) symproms ${ }^{14)}$. Mork and Westgaard also reported that patients with shoulder and neck pain had significantly greater and longer upper trapezius activity than pain-free individuals ${ }^{19)}$. Our study found that the supine position with DHF increased activation of the upper trapezius and scalene muscle on the right side only. The unilateral activations of these muscles caused right lateral flexion of the cervical spine. Unilateral activation of the right trapezius caused left rotation, while that of the scalene caused right rotation ${ }^{14)}$. Therefore, the DHF posture would lead to unbalanced alignment of the cervical spine.

Many studies have investigated the effects of different pillow types on pain intensity, the shape of the cervical spine, and muscle fatigue in patients with cervical spine pain ${ }^{2,8,10)}$. Even when an individual uses the most comfortable pillow, neck muscle activity may differ according to the habitual sleep posture. Therefore, this study suggests that the correct sleep posture is important to prevent musculoskeletal pain of the neck and shoulders.

This study had several limitations. First, all of the participants were healthy and young. Second, this result cannot be generalized because only one type of pillow was used. Third, this study did not measure the actual ribcage and clavicle motion. Fourth, this study assumed that the positions reflected those in sleep, but results of this study may not represent actual sleep conditions. Further studies are required to measure the clavicle and ribcage motion in different sleep postures and the effect of sleep posture on neck muscle activity in subjects with neck and shoulder pain.

In conclusion, the activities of the right trapezius and scalene muscles differed significantly among the three sleeping postures examined and were greatest in the supine position with the dominant hand on the forehead. This study suggests that the correct sleep posture is important and prevent neck and shoulder musculoskeletal pain.

\section{REFERENCES}

1) Foreman MD, Wykle M: Nursing standard-of-practice protocol: sleep disturbances in elderly patients. The NICHE Faculty. Geriatr Nurs, 1995 , $16: 238-243$. [Medline] [CrossRef]

2) Her JG, Ko DH, Woo JH, et al.: Development and comparative evaluation of new shapes of pillows. J Phys Ther Sci, 2014, 26: 377-380. [Medline] [CrossRef]

3) Kyle SD, Morgan K, Espie CA: Insomnia and health-related quality of life. Sleep Med Rev, 2010, 14: 69-82. [Medline] [CrossRef]

4) Roehrs T, Roth T: Sleep and pain: interaction of two vital functions. Semin Neurol, 2005, 25: 106-116. [Medline] [CrossRef]

5) Jeon MY, Jeong H, Lee S, et al.: Improving the quality of sleep with an optimal pillow: a randomized, comparative study. Tohoku J Exp Med, 2014, 233: 183-188. [Medline] [CrossRef]

6) Gordon SJ, Trott P, Grimmer KA: Waking cervical pain and stiffness, headache, scapular or arm pain: gender and age effects. Aust J Physiother, 2002, 48: 9-15. [Medline] [CrossRef]

7) Gordon SJ, Grimmer-Somers KA, Trott PH: Pillow use: the behavior of cervical stiffness, headache and scapular/arm pain. J Pain Res, 2010, 3: 137-145. [Medline]

8) Gordon SJ, Grimmer-Somers K, Trott P: Pillow use: the behaviour of cervical pain, sleep quality and pillow comfort in side sleepers. Man Ther, 2009, 14: 671-678. [Medline] [CrossRef]

9) Liu SF, Lee YL, Liang JC: Shape design of an optimal comfortable pillow based on the analytic hierarchy process method. J Chiropr Med, 2011, 10: 229-239. [Medline] [CrossRef]

10) Lavin RA, Pappagallo M, Kuhlemeier KV: Cervical pain: a comparison of three pillows. Arch Phys Med Rehabil, 1997, 78: 193-198. [Medline] [CrossRef] 
11) Cram J, Kasman G, Holtz J: Introduction of surface electromyography. Gaithersburg: Aspen Publishers, 1988.

12) Neumann DA: Kinesiology of the musculoskeletal system: foundations for physical rehabilitation. St Lous, Mosby: 2002.

13) van der Helm FC, Pronk GM: Three-dimensional recording and description of motions of the shoulder mechanism. J Biomech Eng, 1995, 117: 27-40. [Medline] [CrossRef]

14) Muscolino JE: Know the body: muscle, bone, and palpation essentials. St Louis: Mosby, 2011.

15) Sahrmann SA: Movenemt system impairment syndromes of the extremities, cervical and thoracic spines. St Louis: Elsevier, 2011.

16) Falla D, Jull G, Hodges P, et al.: An endurance-strength training regime is effective in reducing myoelectric manifestations of cervical flexor muscle fatigue in females with chronic neck pain. Clin Neurophysiol, 2006, 117: 828-837. [Medline] [CrossRef]

17) Jull G, Kristjansson E, Dall'Alba P: Impairment in the cervical flexors: a comparison of whiplash and insidious onset neck pain patients. Man Ther, 2004, 9 : 89-94. [Medline] [CrossRef]

18) Dwyer A, Aprill C, Bogduk N: Cervical zygapophyseal joint pain patterns. I: a study in normal volunteers. Spine, 1990, 15: 453-457. [Medline] [CrossRef]

19) Mork PJ, Westgaard RH: The association between nocturnal trapezius muscle activity and shoulder and neck pain. Eur J Appl Physiol, 2004, 92: 18-25. [Medline] [CrossRef] 